\title{
Organization of heart failure care in Spain: characteristics of heart failure units
}

\author{
Alfonso Castro-Beiras, Manuel Anguita-Sánchez, Josep Comín, José Manuel Vázquez- \\ Rodríguez, Trinidad de Frutos, Javier Muñiz
}

\section{To the Editor,}

Heart failure (HF) is a major health problem, and the burden it places on health care systems and society in general has increased in recent years and is expected to continue to grow. ${ }^{1}$ Due to the wide diversity of patients with very different prognoses and therapeutic options, current guidelines recommend a multidisciplinary approach and the establishment of organizational structures to guarantee its implementation (recommendation class I A). ${ }^{2}$

Here we present the results of the MOSAIC (Mapa de la Organización de la Insuficiencia Cardiaca en España [Map of Heart Failure Organization in Spain]) project, which updates and expands the information available about resources and organizational structures in Spain for the care of patients with $\mathrm{HF}^{3}$ and complements the data from other recent studies conducted by the Spanish Society of Cardiology. ${ }^{4}$

We contacted 219 of the 246 hospitals listed (89\%) in the general catalog of the Spanish Ministry of Health, Social Services, and Equality (Ministerio de Sanidad, Servicios Sociales e Igualdad [MSSSI]) ${ }^{5}$; 60 hospitals declined to participate, and 8 were excluded due to insufficient data. We thus present information from 151 hospitals, obtained by an online and telephone survey in the last quarter of 2011.

The hospitals were classified into 3 groups according to the complexity of health care provision: level 1 (no hemodynamic monitoring, electrophysiology, or cardiac surgery), level 2 (hemodynamic monitoring and/or electrophysiology, but no surgery), and level 3 (hemodynamic monitoring, electrophysiology, and surgery).

The study examined 2 types of HF unit. A general unit is a designated hospital service with an assigned manager and a specific protocol for the care of HF patients. An advanced unit has resources for the treatment of critically ill patients, including the management of advanced HF, pretransplant evaluation, hemodynamic monitoring, and ability to recommend implantation of an automated implantable cardioverter defibrillator or cardiac resynchronization device.

The survey included hospitals from all 17 Spanish autonomous regions and Melilla, with a total assigned catchment population of 34.2 million people. Of these centers, 87 (57.6\%) are level 1 hospitals, $33(21.9 \%)$ are level 2, and $31(20.5 \%)$ are level 3, the most complex level. The annual median number [interquartile range] of hospital discharges of patients admitted for all causes was 9220 [4433-20 648], and the annual number of discharges of patients admitted for HF as the main cause was 409 [156-687]. The Figure shows the proportion of centers with an HF unit broken down by unit type and compares these data with the situation in $2006^{3}$ (although that publication did not describe the criteria used to define the hospital type, which may have differed from those used here). Of the 151 centers, $56(37.1 \%)$ have HF units. Of these, 16 are level 1 centers (28.6\% of all units), $14(25 \%)$ are level 2 centers, and $26(46.4 \%)$ are level 3 centers. Heart failure units are currently found in $18.4 \%$ of level 1 centers, $42.4 \%$ of level 2 centers, and $83.9 \%$ of level 3 centers, figures only slightly higher than those for $2006(P>.05$ for all comparisons). Regarding unit type, 26 hospitals have only a general unit $(46.4 \%$ of centers with an HF unit and $17.2 \%$ of the total), 12 have only an advanced unit (21.4\% and 7.9\%), and 18 have units of both types $(32.1 \%$ and $11.9 \%)$. Centers with an HF unit are larger than those without one, have higher numbers of discharges of patients admitted for all causes (median 18906 [7962-30 984] vs 6000 [3 498-13 154]) and for HF (661 [358-1027] vs 251 [121-493]), and also have more cardiologists (17 [9-23] vs 4 [2-9]). Of the 44 general HF units (26 as the only HF unit and 18 in centers that also have advanced units), most $(n=37[84.1 \%])$ are managed by the cardiology service and a few $(n=6[13.6 \%])$ are managed by internal medicine (the corresponding figures for 2006 were $91 \%$ and $9 \%$ ). The cardiology service participates in 42 of the general units $(95.5 \%$ vs $96 \%$ in 2006$)$, internal medicine in 17 (38.6\% vs $11 \%$ in 2006), rehabilitation in 9 (20.5\% vs $9 \%$ in 2006), and geriatrics in 4 (9.1\% vs $22 \%$ in 2006) - the same 
proportion as psychiatry/psychology and social care. Nursing staff are assigned to the general HF unit at all centers except for 1 (compared with $78 \%$ in 2006), but 20 (45.5\%) of the 44 centers reported that no nursing staff are assigned to the unit full-time (compared with a calculated figure of $74 \%$ for 2006). These general units vary greatly in the programs they run, their organization and activities, and the tasks assigned to nursing staff ( Table). In $46.7 \%$ of the advanced HF units (14 of 30), staff do not participate in the follow-up of patients' automated implantable cardioverter defibrillators or cardiac resynchronization devices.

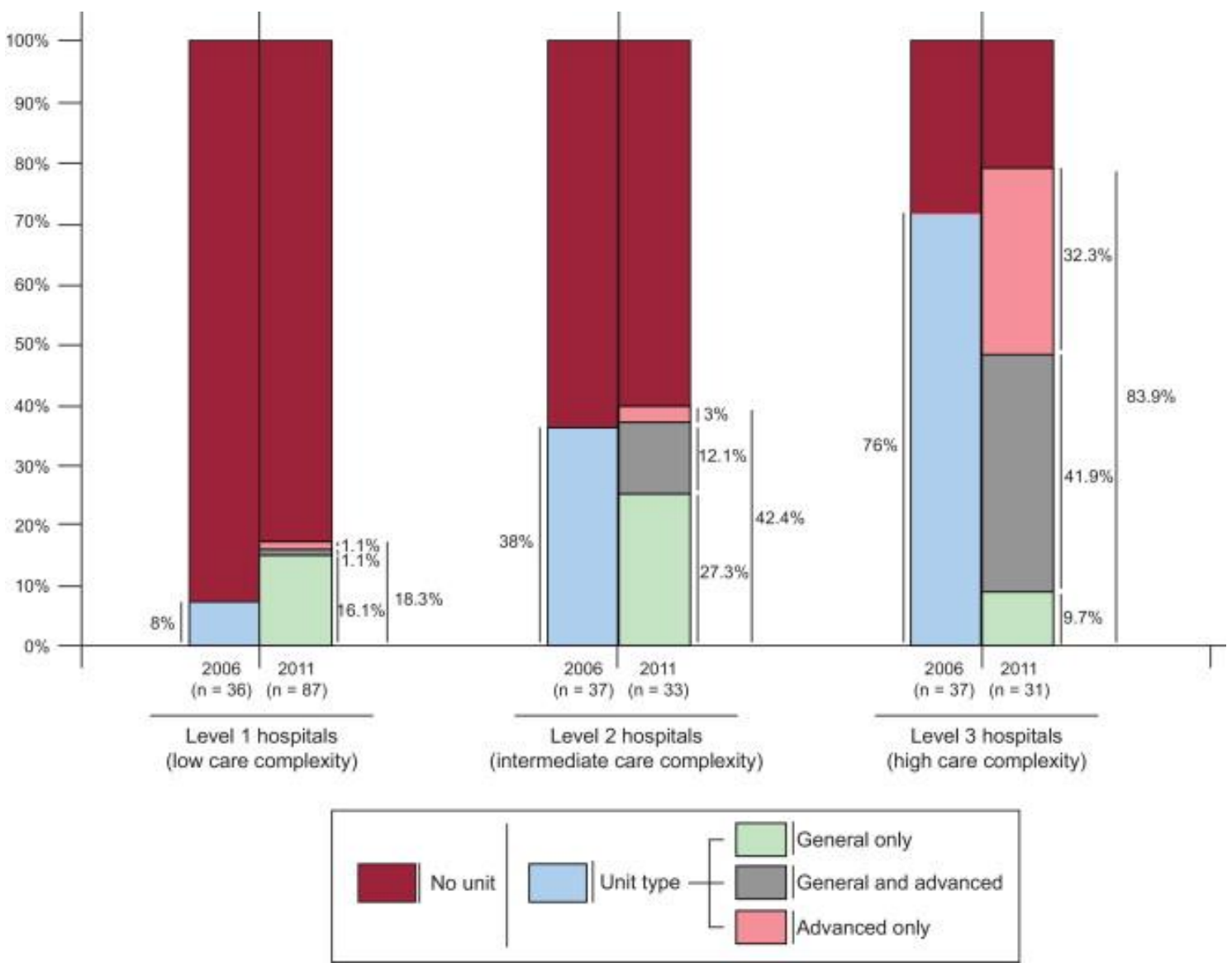

Figure. Heart failure units by type and hospital complexity. 


$\begin{array}{ll}\text { Programs and protocols in the unit } & \\ \text { Cardiac rehabilitation program } & 20(45.5) \\ \text { Joint protocol with internal medicine } & 19(43.2) \\ \text { Joint protocol with primary care } & 21(47.7) \\ \text { Hospital care program } & 10(22.7) \\ \text { Specific telemedicine program } & 9(20.5) \\ \text { Unstructured telephone contact } & 5(11.4) \\ \text { Structured follow-up by telephone- } & 6(13.6) \\ \text { Collection of biometric data with remote telemonitoring devices } & 5(11.4) \\ \text { Remote tele-intervention by video link } & 1(2.3) \\ \text { Remote telemonitoring of implantable devices (AICD and resynchonization devices) }) & 6(13.6) \\ \text { Day-hospital } & 28(63.6) \\ \text { Unit with seating } & 15(34.1) \\ \text { Rotation of resident physicians } & 21(47.7) \\ & \\ \text { Responsibilities of nursing staff } & \\ \text { Training of patients and carers } & \\ \text { Patient care support tasks (eg, ECG) } & 41(93.2) \\ \text { Nurse-led clinic } & 42(95.5) \\ \text { Contacting and assessing patients by telephone } & 29(65.9) \\ \text { Link with primary care physician and nurses } & 35(79.5) \\ \text { Telemonitoring } & 31(70.5) \\ & 11(25)\end{array}$

AICD, automated implantable cardioverter defibrillator; ECG, electrocardiogram.

Values are expressed as no. (\%).

* Following a written protocol and contact schedule.

In summary, the proportion of hospitals with an HF unit has changed little since $2006,{ }^{3}$ indicating ample room for improvement to meet the guideline recommendations, ${ }^{2}$ especially in level 1 centers. Moreover, units with a similar level of technological sophistication vary greatly in their organization and the services provided. There is also ample room for improvement in the role played by advanced HF units, many of which do not currently participate in the follow-up of the devices they have recommended for patients.

Further progress is needed in the specification and establishment of HF units, both general and advanced, while ensuring that they are adapted to local conditions and aim to achieve maximum efficiency with the allocated resources.

\section{FUNDING}

This study was funded by Pfizer S.L.U.

\section{CONFLICTS OF INTEREST}

A. Castro-Beiras, M. Anguita, J. Comín, J.M. Vázquez-Rodríguez and J. Muñiz received financial remuneration from SANED (the company contracted to undertake field work) for advice on the development of this project.

\section{Acknowledgments}

This study is recognized and supported by the Heart Failure and Heart Transplantation Section of the Spanish Society of Cardiology. Field work was conducted by SANED. 


\section{References}

1 I. Sayago-Silva, F. García-López, J. Segovia-Cubero. Epidemiología de la enfermedad cardiovascular en España en los últimos 20 años. Rev Esp Cardiol., 66 (2013), pp. 649-656

2. J.J. McMurray, S. Adamopoulos, S.D. Anker, A. Auricchio, M. Bohm, K. Dickstein, et al. ESC Guidelines for the diagnosis and treatment of acute and chronic heart failure 2012: The Task Force for the Diagnosis and Treatment of Acute and Chronic Heart Failure 2012 of the European Society of Cardiology. Developed in collaboration with the Heart Failure Association (HFA) of the ESC. Eur Heart J., 33 (2012), pp. 1787-1847

3. E. Zamora, J. Lupón. Unidades de insuficiencia cardíaca en España: situación actual. Rev Esp Cardiol., 60 (2007), pp. 874-877

4. Sociedad Española de Cardiología. Registro RECALCAR. La atención al paciente con cardiopatía en el Sistema Nacional de Salud. Recursos, actividad y calidad asistencial. Informe 2013. Available July 7, 2014 at: http://www.secardiologia.es/images/stories//registros/recalcar/Informe-Recalcar-2013.pdf

5. Catálogo nacional de hospitales [Internet]. Madrid: Ministerio de Sanidad, Servicios Sociales e Igualdad; 2006 [accessed May 15, 2011]. Available at:

https://www.msssi.gob.es/ciudadanos/prestaciones/centrosServiciosSNS/hospitales/home.htm 